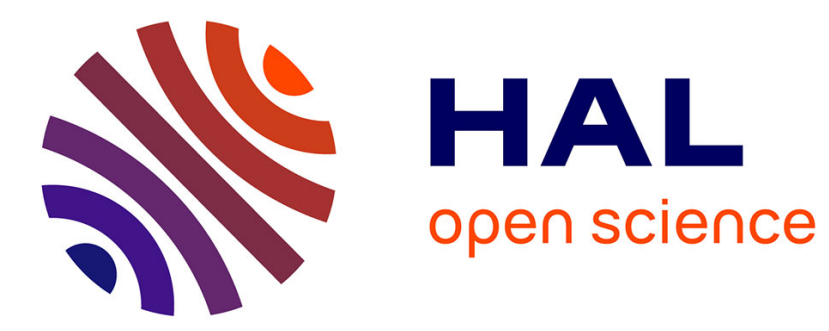

\title{
Bases for spin systems and qudits from angular momentum theory
}

Maurice R. Kibler

\section{To cite this version:}

Maurice R. Kibler. Bases for spin systems and qudits from angular momentum theory. Communications in Nonlinear Science and Numerical Simulation, 2010, 15, pp.752-763. 10.1016/j.cnsns.2009.05.017 . in2p3-00333758

\section{HAL Id: in2p3-00333758 https://hal.in2p3.fr/in2p3-00333758}

Submitted on 24 Oct 2008

HAL is a multi-disciplinary open access archive for the deposit and dissemination of scientific research documents, whether they are published or not. The documents may come from teaching and research institutions in France or abroad, or from public or private research centers.
L'archive ouverte pluridisciplinaire HAL, est destinée au dépôt et à la diffusion de documents scientifiques de niveau recherche, publiés ou non, émanant des établissements d'enseignement et de recherche français ou étrangers, des laboratoires publics ou privés. 


\title{
Bases for spin systems and qudits from angular momentum theory
}

\author{
Maurice R. Kibler*1,2 \\ ${ }^{*}{ }^{1}$ Université de Lyon, F-69622, Lyon, France ${ }^{2}$ Université Lyon 1, \\ Villeurbanne, CNRS/IN2P3, UMR5822, Institut de Physique \\ Nucléaire de Lyon (e-mail: kibler@ipnl.in2p3.fr)
}

\begin{abstract}
Spin bases of relevance for quantum systems with cyclic symmetry as well as for quantum information and quantum computation are constructed from the theory of angular momentum. This approach is connected to the use of generalized Pauli matrices (in dimension d) arising from a polar decomposition of the group $\mathrm{SU}_{2}$. Numerous examples are given for $d=2,3$ and 4 .
\end{abstract}

Keywords: Spin systems, qubits, qudits, generalized Pauli matrices, mutually unbiased bases, generalized Pauli group.

\section{INTRODUCTION}

Hilbert spaces of finite dimension $d$ play an important role for the quantum mechanical description of dynamical systems and systems of qudits (qubits correspond to $d=2$, qudits to $d$ arbitrary). Generally speaking, bases for finitedimensional subspaces of the representation space of the group $\mathrm{SO}_{3}$ or its universal covering $\mathrm{SU}_{2}$ can be used for spin systems (used in spectroscopy and quantum chemistry) and qudits (used in quantum information and quantum computation). Such bases can be constructed from tools developed for a study of supersymmetric quantum mechanics [1-4] and for a nonstandard approach to the representations of $\mathrm{SU}_{2}[5-7]$.

It is the object of this talk to describe a method for constructing bases which are of central importance in quantum information theory, namely, mutually unbiased bases (MUBs). In dimension $d$, two bases are said to be unbiased if and only if the modulus of the inner product of any vector of one basis with any vector of the other one is equal $1 / \sqrt{d}[8-17]$. These bases are of paramount importance for quantum cryptography and quantum state tomography.

We develop here an approach that gives a complete solution for the construction of MUBs in the case where the dimension $d$ of the considered Hilbert space is a prime number. This approach is based on a nonstandard approach to the theory of angular momentum viewed through the prism of the Lie algebra of $\mathrm{SU}_{2}$. The concepts of Weyl pairs and generalized Pauli group useful in quantum computation and quantum information are also briefly discussed.

Most of the material presented in this work takes its origin in papers published by the author and its collaborators [18-26].

The notation adopted here is the one used in quantum mechanics. Let us simply mention that $\delta_{a, b}$ stands for the Kronecker symbol for $a$ and $b, A^{\dagger}$ denotes the adjoint of the operator $A$, and $[A, B]$ is the commutator of the operators $A$ and $B$.

\section{SPIN BASES}

\subsection{Angular momentum states}

Let us consider a generalized angular momentum. We denote as $j^{2}$ and $j_{z}$ its square and $z$-component, respectively. The common orthonormalized eigenvectors of the two commuting operators $j^{2}$ and $j_{z}$ are written $|j, m\rangle$. We know that

$$
j^{2}|j, m\rangle=j(j+1)|j, m\rangle, \quad j_{z}|j, m\rangle=m|j, m\rangle,
$$

where $m=j, j-1, \ldots,-j$ and $2 j \in \mathbf{N}$. For a fixed value of the quantum number $j$, we note $\mathcal{E}(2 j+1) \sim \mathbf{C}^{2 j+1}$ the $(2 j+1)$-dimensional Hilbert space spanned by the basis

$$
b_{s}=\{|j, m\rangle: m=j, j-1, \ldots,-j\} .
$$

The basis $b_{s}$ is adapted to spherical symmetry (adapted to the group $\mathrm{SO}_{3}$ if $j \in \mathbf{N}$ or $\mathrm{SU}_{2}$ if $2 j \in \mathbf{N}$ ).

In the applications to spectroscopy, the generalized angular momentum can be an orbital angular momentum, a spin angular momentum, a total (spin + orbital) angular momentum, a nucleus spin, etc. The vectors $|j, m\rangle$ can thus have several realizations. For example, in the spectroscopy of a partly filled shell ion with configuration $n \ell^{N}$, we have state vectors of type $|J, M\rangle \equiv\left|n \ell^{N} \tau S L J M\right\rangle$ in the Russell-Saunders coupling (here $j=J$ and $m=M$ ).

The vectors $|j, m\rangle$ can also serve for quantum information and quantum computation. By introducing the notation

$$
k=j-m, \quad|k\rangle=|j, m\rangle, \quad d=2 j+1,
$$

we get the vectors $|0\rangle$ (for $m=j),|1\rangle$ (for $m=j-1$ ), . , $|d-1\rangle$ (for $m=-j$ ). The vectors $|0\rangle,|1\rangle, \ldots,|d-1\rangle$ are called qudits. Then, the basis $b_{s}$ becomes 


$$
B_{d}=\{|k\rangle: k=0,1, \ldots, d-1\},
$$

which is referred to as the computational basis in quantum information theory. In $d$ dimensions, the most general qudit is written as

$$
c_{0}|0\rangle+c_{1}|1\rangle+\ldots+c_{d-1}|d-1\rangle,
$$

where $c_{k} \in \mathbf{C}$ for $k=0,1, \ldots, d-1$. The case $d=2 \Leftrightarrow$ $j=1 / 2$ corresponds to qubits generated by $|0\rangle=\left|\frac{1}{2}, \frac{1}{2}\right\rangle$ and $|1\rangle=\left|\frac{1}{2},-\frac{1}{2}\right\rangle$. Note that the vectors $\left|\frac{1}{2}, \frac{1}{2}\right\rangle$ and $\left|\frac{1}{2},-\frac{1}{2}\right\rangle$ are nothing but the spinorbitals $\alpha$ (for spin up) and $\beta$ (for spin down), respectively, used in quantum chemistry.

In the rest of this paper, we shall use both the notation $|k\rangle$ familiar in (i) quantum information and quantum computation and (ii) the description of cyclic systems (for which $|d\rangle \equiv|0\rangle,|d+1\rangle \equiv|1\rangle, \ldots)$ and equally well the notation $|j, m\rangle$ employed in (i) angular momentum theory (from a physical point of view or in its approach from the group $\mathrm{SU}_{2}$ ), (ii) nuclear, atomic and molecular spectroscopy, and (iii) quantum chemistry. For $d=2$, we shall play with the notation

$$
\alpha=\left|\frac{1}{2}, \frac{1}{2}\right\rangle=|0\rangle
$$

and

$$
\beta=\left|\frac{1}{2},-\frac{1}{2}\right\rangle=|1\rangle
$$

\subsection{A noncanonical basis for $\mathrm{SU}_{2}$}

We now define the operator $v_{0 a}$ (a particular case of the unitary operator $v_{r a}$ introduced in $\left.[21,22]\right)$ through

$$
v_{0 a}|j, m\rangle=\left(1-\delta_{m, j}\right) q^{(j-m) a}|j, m+1\rangle+\delta_{m, j}|j,-j\rangle
$$

or equivalently

$$
v_{0 a}|k\rangle=q^{k a}|k-1\rangle
$$

where $a=0,1, \ldots, 2 j=d-1, q=\exp (2 \pi i / d)$, and $k-1$ should be understood modulo $d$ (i.e., $|-1\rangle=|d-1\rangle$ ). It is evident that the operators $j^{2}$ and $v_{0 a}$ commute so that the (complete) set $\left\{j^{2}, v_{0 a}\right\}$ constitutes an alternative to the set $\left\{j^{2}, j_{z}\right\}[5-7,18]$. The matrix $V_{0 a}$ of the linear operator $v_{0 a}$ on the basis $b_{s} \equiv B_{d}$ reads

$$
V_{0 a}=\left(\begin{array}{ccccc}
0 & q^{a} & 0 & \ldots & 0 \\
0 & 0 & q^{2 a} & \ldots & 0 \\
\vdots & \vdots & \vdots & \ldots & \vdots \\
0 & 0 & 0 & \ldots & q^{2 j a} \\
1 & 0 & 0 & \ldots & 0
\end{array}\right)
$$

where the $d(=2 j+1)$ lines and $d$ columns are labeled in the order $|0\rangle=|j, j\rangle,|1\rangle=|j, j-1\rangle, \ldots,|d-1\rangle=|j,-j\rangle$.

By introducing the Hermitian operator $h$ via

$$
h|j, m\rangle=\sqrt{(j+m)(j-m+1)}|j, m\rangle
$$

or

$$
h|k\rangle=\sqrt{(d-1-k)(k+1)}|k\rangle,
$$

it can be shown that the three operators

$$
j_{+}=h v_{0 a}, \quad j_{-}=v_{0 a}^{\dagger} h, \quad j_{z}=\frac{1}{2}\left(h^{2}-v_{0 a}^{\dagger} h^{2} v_{0 a}\right)
$$

satisfy the commutation relations

$$
\left[j_{z}, j_{+}\right]=+j_{+}, \quad\left[j_{z}, j_{-}\right]=-j_{-}, \quad\left[j_{+}, j_{-}\right]=2 j_{z} .
$$

Therefore, $j_{+}, j_{-}$and $j_{z}$ span the Lie algebra $\mathrm{su}_{2}$ of $\mathrm{SU}_{2}$ and the operators $v_{0 a}$ and $h$ realize a polar decomposition of $\mathrm{su}_{2}[5-7,18-19,21-22]$.

We may ask what are the analogues of the vectors $|j, m\rangle$ in the $\left\{j^{2}, v_{0 a}\right\}$ scheme? In fact, the common eigenvectors of the commuting operators $v_{0 a}$ and $j^{2}$ are [18-19,21-22]

$$
|a \alpha\rangle=\frac{1}{\sqrt{2 j+1}} \sum_{m=-j}^{j} q^{(j+m)(j-m+1) a / 2+(j+m) \alpha}|j, m\rangle
$$

or alternatively

$$
|a \alpha\rangle=\frac{1}{\sqrt{d}} \sum_{k=0}^{d-1} q^{(d-k-1)(k+1) a / 2-(k+1) \alpha}|k\rangle,
$$

where $\alpha$ can take the values $\alpha=0,1, \ldots, 2 j=d-1$. These vectors satisfy the eigenvalue equation

$$
v_{0 a}|a \alpha\rangle=q^{j a-\alpha}|a \alpha\rangle=q^{(d-1) a / 2-\alpha}|a \alpha\rangle
$$

that corresponds to a nondegenerate spectrum for the operator $v_{0 a}$. For fixed $j$ and $a$ (with $2 j \in \mathbf{N}$ and $a \in \mathbf{Z}_{d}$ ), the set

$$
B_{0 a}=\{|a \alpha\rangle: \alpha=0,1, \ldots, d-1\}
$$

is an orthonormal basis for $\mathcal{E}(d)$, which is an alternative to the basis $B_{d}$.

\section{APPLICATION TO QUANTUM INFORMATION}

We are now in a position to discuss some results of interest for quantum information.

\subsection{Weyl pairs}

Besides the operator $v_{0 a}$, it is interesting to define the unitary operator $z$ through

$$
z=\left(v_{00}\right)^{\dagger} v_{01}
$$

This definition yields

$$
z|j, m\rangle=q^{j-m}|j, m\rangle \Leftrightarrow z|k\rangle=q^{k}|k\rangle .
$$

Therefore, we have the shift property

$$
z|a \alpha\rangle=q^{-1}\left|a \alpha_{1}\right\rangle, \quad \alpha_{1}=\alpha-1
$$

Furthermore, we can show that $v_{0 a}$ is connected to $z$ and $x=v_{00}$ by 


$$
v_{0 a}=x z^{a}
$$

The two isospectral operators $x$ (a shift operator when acting on $|j, m\rangle$ and a phase operator when acting on $|a \alpha\rangle$ ) and $z$ (a phase operator when acting on $|j, m\rangle$ and a shift operator when acting on $|a \alpha\rangle)$ are often called shift operator and clock operator, respectively, in quantum information and quantum computation. Note that for each of the operators $x$ and $z$, the shift or clock character depends on which state, $|j, m\rangle=|k\rangle$ or $|a \alpha\rangle$, the operator acts. The operators $v_{0 a}$ and $z$ satisfy

$$
e^{-i \pi(d-1) a}\left(v_{0 a}\right)^{d}=z^{d}=1, \quad v_{0 a} z-q z v_{0 a}=0 .
$$

In other words, the unitary operators $v_{0 a}$ and $z$ are cyclic (up to a phase factor for $v_{0 a}$ with $a \neq 0$ ) and do not commute. The pair $(x, z)$ corresponding to $a=0$ is a Weyl pair in the sense that $x^{d}=z^{d}=1$ and $x z=q z x$. Such a pair can be used as an integrity basis for constructing the Lie algebra of the unitary group $\mathrm{U}_{d}[24]$.

In the basis $B_{d}$, the $d$-dimensional matrices $X$ and $Z$ of the linear operators $x$ and $z$ are given by

$$
X=\left(\begin{array}{ccccc}
0 & 1 & 0 & \ldots & 0 \\
0 & 0 & 1 & \ldots & 0 \\
\vdots & \vdots & \vdots & \ldots & \vdots \\
0 & 0 & 0 & \ldots & 1 \\
1 & 0 & 0 & \ldots & 0
\end{array}\right)
$$

and

$$
Z=\left(\begin{array}{ccccc}
1 & 0 & 0 & \ldots & 0 \\
0 & q & 0 & \ldots & 0 \\
0 & 0 & q^{2} & \ldots & 0 \\
\vdots & \vdots & \vdots & \ldots & \vdots \\
0 & 0 & 0 & \ldots & q^{d-1}
\end{array}\right)
$$

For $d=2 \Leftrightarrow j=1 / 2(\Rightarrow q=-1)$, the matrices $V_{00}, V_{01}$, and $Z$ are connected to the Pauli matrices $\sigma_{x}, \sigma_{y}$, and $\sigma_{z}$ via

$$
V_{00}=X=\sigma_{x}, \quad V_{01}=X Z=-i \sigma_{y}, \quad Z=\sigma_{z} .
$$

For $d$ arbitrary, the pair $\left(X=V_{00}, Z=V_{00}^{\dagger} V_{01}\right)$ are basic ingredients for generating generalized Pauli matrices and a generalized Pauli group $P_{d}$ of interest in quantum computation for quantum correcting codes [24,25].

\subsection{Mutually unbiased bases}

We now give two applications to MUBs.

First, in the general case where $d$ is arbitrary, we can check that

$$
|\langle k \mid a \alpha\rangle|=\frac{1}{\sqrt{d}}, \quad k, a, \alpha \in \mathbf{Z}_{d}
$$

and

$$
|\langle 0 \alpha \mid 1 \beta\rangle|=\frac{1}{\sqrt{d}}, \quad \alpha, \beta \in \mathbf{Z}_{d}
$$

where we use $\langle\mid\rangle$ to denote the inner product in $\mathcal{E}(d)$. In the terminology of quantum information, the latter two equations mean that the bases $B_{d}, B_{00}$, and $B_{01}$ are three MUBs. This result is in agreement with the one according to which there exist at least three MUBs in arbitrary dimension, see for instance [17].

Second, in the special case where $d=p$ is a prime number, the latter equation can be extended to

$$
|\langle a \alpha \mid b \beta\rangle|=\delta_{\alpha, \beta} \delta_{a, b}+\frac{1}{\sqrt{p}}\left(1-\delta_{a, b}\right), \quad a, b, \alpha, \beta \in \mathbf{Z}_{d}
$$

The proof follows from the use of generalized quadratic Gauss sums [21]. Hence, the bases $B_{00}, B_{01}, \ldots, B_{0 p-1}$, and $B_{p}$ constitute a (maximal) set of $p+1$ MUBs. This result is a particular case of the one according to which there exist a maximal set of $d+1$ MUBs when $d$ is the power of a prime number [8-17].

We continue with some typical examples of interest for quantum information and quantum computation.

\section{EXAMPLES}

\subsection{The case $d=2$}

In this case, relevant for a spin $j=1 / 2$ or for a qubit, we have $q=-1$ and $a, \alpha \in \mathbf{Z}_{2}$. The matrices of the operators $v_{0 a}$ are

$$
V_{00}=\left(\begin{array}{ll}
0 & 1 \\
1 & 0
\end{array}\right)=\sigma_{x}, \quad V_{01}=\left(\begin{array}{cc}
0 & -1 \\
1 & 0
\end{array}\right)=-i \sigma_{y} .
$$

The $d+1=3$ MUBs $B_{2}, B_{00}$, and $B_{01}$ are the following. The $B_{2}$ basis:

$$
|0\rangle, \quad|1\rangle .
$$

The $B_{00}$ basis:

$$
|00\rangle=\frac{1}{\sqrt{2}}(|0\rangle+|1\rangle), \quad|01\rangle=\frac{1}{\sqrt{2}}(-|0\rangle+|1\rangle) .
$$

The $B_{01}$ basis:

$$
|10\rangle=\frac{1}{\sqrt{2}}(i|0\rangle+|1\rangle), \quad|11\rangle=\frac{1}{\sqrt{2}}(-i|0\rangle+|1\rangle) .
$$

or, by using the spinorbitals $\alpha$ and $\beta$, we get

The $B_{2}$ basis:

$$
\alpha, \quad \beta
$$

The $B_{00}$ basis:

$$
|00\rangle=\frac{1}{\sqrt{2}}(\alpha+\beta), \quad|01\rangle=-\frac{1}{\sqrt{2}}(\alpha-\beta) .
$$

The $B_{01}$ basis:

$$
|10\rangle=i \frac{1}{\sqrt{2}}(\alpha-i \beta), \quad|11\rangle=-i \frac{1}{\sqrt{2}}(\alpha+i \beta) .
$$

In terms of eigenvectors of the matrices $V_{0 a}$, we must replace the state vectors $|a \alpha\rangle$ by column vectors. This leads to 
The $B_{2}$ basis:

$$
\alpha \rightarrow\left(\begin{array}{l}
1 \\
0
\end{array}\right), \quad \beta \rightarrow\left(\begin{array}{l}
0 \\
1
\end{array}\right)
$$

The $B_{00}$ basis:

$$
|00\rangle \rightarrow \frac{1}{\sqrt{2}}\left(\begin{array}{l}
1 \\
1
\end{array}\right), \quad|01\rangle \rightarrow-\frac{1}{\sqrt{2}}\left(\begin{array}{c}
1 \\
-1
\end{array}\right) .
$$

The $B_{01}$ basis:

$$
|10\rangle \rightarrow i \frac{1}{\sqrt{2}}\left(\begin{array}{c}
1 \\
-i
\end{array}\right), \quad|11\rangle \rightarrow-i \frac{1}{\sqrt{2}}\left(\begin{array}{l}
1 \\
i
\end{array}\right) .
$$

\subsection{The case $d=3$}

This case corresponds to a spin $j=1$ or to a qutrit. Here, we have $q=\exp (2 \pi i / 3)$ and $a, \alpha \in \mathbf{Z}_{3}$. The matrices of the operators $v_{0 a}$ are

$$
\begin{aligned}
V_{00} & =\left(\begin{array}{lll}
0 & 1 & 0 \\
0 & 0 & 1 \\
1 & 0 & 0
\end{array}\right) \\
V_{01} & =\left(\begin{array}{lll}
0 & q & 0 \\
0 & 0 & q^{2} \\
1 & 0 & 0
\end{array}\right) \\
V_{02} & =\left(\begin{array}{lll}
0 & q^{2} & 0 \\
0 & 0 & q \\
1 & 0 & 0
\end{array}\right) .
\end{aligned}
$$

The $\mathrm{d}+1=4$ MUBs $B_{3}, B_{00}, B_{01}$, and $B_{02}$ are the following.

The $B_{3}$ basis:

$$
|0\rangle, \quad|1\rangle, \quad|2\rangle
$$

The $B_{00}$ basis:

$$
\begin{aligned}
& |00\rangle=\frac{1}{\sqrt{3}}(|0\rangle+|1\rangle+|2\rangle) \\
& |01\rangle=\frac{1}{\sqrt{3}}\left(q^{2}|0\rangle+q|1\rangle+|2\rangle\right) \\
& |02\rangle=\frac{1}{\sqrt{3}}\left(q|0\rangle+q^{2}|1\rangle+|2\rangle\right) .
\end{aligned}
$$

The $B_{01}$ basis:

$$
\begin{aligned}
& |10\rangle=\frac{1}{\sqrt{3}}(q|0\rangle+q|1\rangle+|2\rangle) \\
& |11\rangle=\frac{1}{\sqrt{3}}\left(|0\rangle+q^{2}|1\rangle+|2\rangle\right) \\
& |12\rangle=\frac{1}{\sqrt{3}}\left(q^{2}|0\rangle+|1\rangle+|2\rangle\right) .
\end{aligned}
$$

The $B_{02}$ basis:

$$
\begin{aligned}
& |20\rangle=\frac{1}{\sqrt{3}}\left(q^{2}|0\rangle+q^{2}|1\rangle+|2\rangle\right) \\
& |21\rangle=\frac{1}{\sqrt{3}}(q|0\rangle+|1\rangle+|2\rangle) \\
& |22\rangle=\frac{1}{\sqrt{3}}(|0\rangle+q|1\rangle+|2\rangle) .
\end{aligned}
$$

This can be transcribed in terms of column vectors as follows.

The $B_{3}$ basis:

$$
|0\rangle \rightarrow\left(\begin{array}{l}
1 \\
0 \\
0
\end{array}\right), \quad|1\rangle \rightarrow\left(\begin{array}{l}
0 \\
1 \\
0
\end{array}\right), \quad|2\rangle \rightarrow\left(\begin{array}{l}
0 \\
0 \\
1
\end{array}\right)
$$

The $B_{00}$ basis:

$|00\rangle \rightarrow \frac{1}{\sqrt{3}}\left(\begin{array}{l}1 \\ 1 \\ 1\end{array}\right),|01\rangle \rightarrow \frac{1}{\sqrt{3}}\left(\begin{array}{c}q^{2} \\ q \\ 1\end{array}\right),|02\rangle \rightarrow \frac{1}{\sqrt{3}}\left(\begin{array}{c}q \\ q^{2} \\ 1\end{array}\right)$

The $B_{01}$ basis:

$$
|10\rangle \rightarrow \frac{1}{\sqrt{3}}\left(\begin{array}{c}
q \\
q \\
1
\end{array}\right),|11\rangle \rightarrow \frac{1}{\sqrt{3}}\left(\begin{array}{c}
1 \\
q^{2} \\
1
\end{array}\right),|12\rangle \rightarrow \frac{1}{\sqrt{3}}\left(\begin{array}{c}
q^{2} \\
1 \\
1
\end{array}\right)
$$

The $B_{02}$ basis:

$$
|20\rangle \rightarrow \frac{1}{\sqrt{3}}\left(\begin{array}{c}
q^{2} \\
q^{2} \\
1
\end{array}\right),|21\rangle \rightarrow \frac{1}{\sqrt{3}}\left(\begin{array}{c}
q \\
1 \\
1
\end{array}\right),|22\rangle \rightarrow \frac{1}{\sqrt{3}}\left(\begin{array}{c}
1 \\
q \\
1
\end{array}\right)
$$

\subsection{The case $d=4$}

This case corresponds to a spin $j=3 / 2$. Here, we have $q=i$ and $a, \alpha \in \mathbf{Z}_{4}$. The five bases $B_{4}, B_{00}, B_{01}, B_{02}$, and $B_{03}$ do not constitute a system of MUBs $(d=4$ is not a prime number). Nevertheless, it is possible to find $d+1=5$ MUBs because $d=2^{2}$ is the power of a prime number. This can be achieved by replacing the space $\mathcal{E}(4)$ spanned by $\{|3 / 2, m\rangle: m=3 / 2,1 / 2,-1 / 2,-3 / 2\}$ by the tensor product space $\mathcal{E}(2) \otimes \mathcal{E}(2)$ spanned by the canonical (or computational) basis

$$
\{\alpha \otimes \alpha, \alpha \otimes \beta, \beta \otimes \alpha, \beta \otimes \beta\} .
$$

The space $\mathcal{E}(2) \otimes \mathcal{E}(2)$ is associated with the coupling of two spin angular momenta $j_{1}=1 / 2$ and $j_{2}=1 / 2$ or two qubits (in the vector $u \otimes v, u$ and $v$ correspond to $j_{1}$ and $j_{2}$, respectively). An alternative basis for $\mathcal{E}(2) \otimes \mathcal{E}(2)$ is the $\mathrm{SU}_{2}$ adapted basis

$$
\left\{\alpha \otimes \alpha, \frac{1}{2}(\alpha \otimes \beta+\beta \otimes \alpha), \beta \otimes \beta, \frac{1}{2}(\alpha \otimes \beta-\beta \otimes \alpha)\right\},
$$

the vectors of which are well-known in the treatment of spin systems. Such a basis corresponds to the decomposition

$$
(1 / 2) \otimes(1 / 2)=(1) \oplus(0)
$$

in terms of irreducible representation classes of $\mathrm{SU}_{2}$. In the $\mathrm{SU}_{2}$ adapted basis, the first three vectors are symmetric under the interchange $1 \leftrightarrow 2$ and describe a total angular momentum $J=1$ while the last one is antisymmetric and corresponds to $J=0$. It should be observed that the $\mathrm{SU}_{2}$ adapted basis illustrates a connection between the special unitary group $\mathrm{SU}_{2}$ and the permutation group $\mathrm{S}_{2}$ (a particular case of the Schur-Weyl duality theorem between 
irreducible representation classes of the linear group $\mathrm{GL}_{d}$ and the symmetric group $\mathrm{S}_{n}$ ).

In addition to the computational or canonical basis and the $\mathrm{SU}_{2}$ adapted basis, it is possible to find other bases of $\mathcal{E}(2) \otimes \mathcal{E}(2)$ which are mutually unbiased. As a matter of fact, $d=4$ MUBs, besides the computational basis $\{\alpha \otimes \alpha, \alpha \otimes \beta, \beta \otimes \alpha, \beta \otimes \beta\}$, correspond to the eigenvectors

$$
|a b \alpha \beta\rangle=|a \alpha\rangle \otimes|b \beta\rangle
$$

of the operators $w_{a b}=v_{0 a} \otimes v_{0 b}$ (the vectors $|a \alpha\rangle$ and $|b \beta\rangle$ refer to the two spaces $\mathcal{E}(2))$. As a result, we have the $d+1=5$ following MUBs where $\lambda=(1-i) / 2$ and $\mu=(1+i) / 2$.

The canonical basis:

$$
\alpha \otimes \alpha, \quad \alpha \otimes \beta, \quad \beta \otimes \alpha, \quad \beta \otimes \beta
$$

or in column vectors

$$
\left(\begin{array}{l}
1 \\
0 \\
0 \\
0
\end{array}\right), \quad\left(\begin{array}{l}
0 \\
1 \\
0 \\
0
\end{array}\right), \quad\left(\begin{array}{l}
0 \\
0 \\
1 \\
0
\end{array}\right), \quad\left(\begin{array}{l}
0 \\
0 \\
0 \\
1
\end{array}\right) .
$$

The $w_{00}$ basis:

$$
\begin{aligned}
|0000\rangle & =\frac{1}{2}(\alpha \otimes \alpha+\alpha \otimes \beta+\beta \otimes \alpha+\beta \otimes \beta) \\
|0001\rangle & =\frac{1}{2}(\alpha \otimes \alpha-\alpha \otimes \beta+\beta \otimes \alpha-\beta \otimes \beta) \\
|0010\rangle & =\frac{1}{2}(\alpha \otimes \alpha+\alpha \otimes \beta-\beta \otimes \alpha-\beta \otimes \beta) \\
|0011\rangle & =\frac{1}{2}(\alpha \otimes \alpha-\alpha \otimes \beta-\beta \otimes \alpha+\beta \otimes \beta)
\end{aligned}
$$

or in column vectors

$$
\frac{1}{2}\left(\begin{array}{l}
1 \\
1 \\
1 \\
1
\end{array}\right), \quad \frac{1}{2}\left(\begin{array}{c}
1 \\
-1 \\
1 \\
-1
\end{array}\right), \quad \frac{1}{2}\left(\begin{array}{c}
1 \\
1 \\
-1 \\
-1
\end{array}\right), \quad \frac{1}{2}\left(\begin{array}{c}
1 \\
-1 \\
-1 \\
1
\end{array}\right) .
$$

The $w_{11}$ basis:

$$
\begin{aligned}
& |1100\rangle=\frac{1}{2}(\alpha \otimes \alpha+i \alpha \otimes \beta+i \beta \otimes \alpha-\beta \otimes \beta) \\
& |1101\rangle=\frac{1}{2}(\alpha \otimes \alpha-i \alpha \otimes \beta+i \beta \otimes \alpha+\beta \otimes \beta) \\
& |1110\rangle=\frac{1}{2}(\alpha \otimes \alpha+i \alpha \otimes \beta-i \beta \otimes \alpha+\beta \otimes \beta) \\
& |1111\rangle=\frac{1}{2}(\alpha \otimes \alpha-i \alpha \otimes \beta-i \beta \otimes \alpha-\beta \otimes \beta)
\end{aligned}
$$

or in column vectors

$$
\frac{1}{2}\left(\begin{array}{c}
1 \\
i \\
i \\
-1
\end{array}\right), \quad \frac{1}{2}\left(\begin{array}{c}
1 \\
-i \\
i \\
1
\end{array}\right), \quad \frac{1}{2}\left(\begin{array}{c}
1 \\
i \\
-i \\
1
\end{array}\right), \quad \frac{1}{2}\left(\begin{array}{c}
1 \\
-i \\
-i \\
-1
\end{array}\right) .
$$

The $w_{01}$ basis:

$$
\begin{aligned}
& \lambda|0100\rangle+\mu|0111\rangle=\frac{1}{2}(\alpha \otimes \alpha+\alpha \otimes \beta-i \beta \otimes \alpha+i \beta \otimes \beta) \\
& \mu|0100\rangle+\lambda|0111\rangle=\frac{1}{2}(\alpha \otimes \alpha-\alpha \otimes \beta+i \beta \otimes \alpha+i \beta \otimes \beta) \\
& \lambda|0101\rangle+\mu|0110\rangle=\frac{1}{2}(\alpha \otimes \alpha-\alpha \otimes \beta-i \beta \otimes \alpha-i \beta \otimes \beta) \\
& \mu|0101\rangle+\lambda|0110\rangle=\frac{1}{2}(\alpha \otimes \alpha+\alpha \otimes \beta+i \beta \otimes \alpha-i \beta \otimes \beta)
\end{aligned}
$$

or in column vectors

$$
\frac{1}{2}\left(\begin{array}{c}
1 \\
1 \\
-i \\
i
\end{array}\right), \quad \frac{1}{2}\left(\begin{array}{c}
1 \\
-1 \\
i \\
i
\end{array}\right), \quad \frac{1}{2}\left(\begin{array}{c}
1 \\
-1 \\
-i \\
-i
\end{array}\right), \quad \frac{1}{2}\left(\begin{array}{c}
1 \\
1 \\
i \\
-i
\end{array}\right) .
$$

The $w_{10}$ basis:

$$
\begin{aligned}
& \lambda|1000\rangle+\mu|1011\rangle=\frac{1}{2}(\alpha \otimes \alpha-i \alpha \otimes \beta+\beta \otimes \alpha+i \beta \otimes \beta) \\
& \mu|1000\rangle+\lambda|1011\rangle=\frac{1}{2}(\alpha \otimes \alpha+i \alpha \otimes \beta-\beta \otimes \alpha+i \beta \otimes \beta) \\
& \lambda|1001\rangle+\mu|1010\rangle=\frac{1}{2}(\alpha \otimes \alpha+i \alpha \otimes \beta+\beta \otimes \alpha-i \beta \otimes \beta) \\
& \mu|1001\rangle+\lambda|1010\rangle=\frac{1}{2}(\alpha \otimes \alpha-i \alpha \otimes \beta-\beta \otimes \alpha-i \beta \otimes \beta)
\end{aligned}
$$

or in column vectors

$$
\frac{1}{2}\left(\begin{array}{c}
1 \\
-i \\
1 \\
i
\end{array}\right), \quad \frac{1}{2}\left(\begin{array}{c}
1 \\
i \\
-1 \\
i
\end{array}\right), \quad \frac{1}{2}\left(\begin{array}{c}
1 \\
i \\
1 \\
-i
\end{array}\right), \quad \frac{1}{2}\left(\begin{array}{c}
1 \\
-i \\
-1 \\
-i
\end{array}\right) .
$$

It is to be noted that the vectors of the $w_{00}$ and $w_{11}$ bases are not intricated (i.e., each vector is the direct product of two vectors) while the vectors of the $w_{01}$ and $w_{10}$ bases are intricated (i.e., each vector is not the direct product of two vectors).

To be more precise, the degree of intrication of the state vectors for the bases $w_{00}, w_{11}, w_{01}$, and $w_{10}$ can be determined in the following way. In arbitrary dimension $d$, let

$$
|\Phi\rangle=\sum_{k=0}^{d-1} \sum_{l=0}^{d-1} a_{k l}|k\rangle \otimes|l\rangle
$$

be a double qudit state vector. Then, it can be shown that the determinant of the $d \times d$ matrix $A=\left(a_{k l}\right)$ satisfies

$$
0 \leq|\operatorname{det} A| \leq \frac{1}{\sqrt{d^{d}}}
$$

as proved in the Albouy thesis [26]. The case $\operatorname{det} A=0$ corresponds to the absence of global intrication while the case

$$
|\operatorname{det} A|=\frac{1}{\sqrt{d^{d}}}
$$

corresponds to a maximal intrication. As an illustration, we obtain that all the state vectors for $w_{00}$ and $w_{11}$ are not intricated and that all the state vectors for $w_{01}$ and $w_{10}$ are maximally intricated. 


\section{CONCLUSION}

We presented some results concerning bases of interest for spin systems and quantum information. It should be noted that the bases derived in the present work are adapted to cyclic symmetry and can be used for describing finite or infinite cyclic systems. As an extension of this paper and of its companion paper [24], it would be interesting to examine when $d$ is a composite number how the Weyl pair $(X, Z)$ and the corresponding generalized Pauli group $P_{d}$ are connected to the Weyl pairs and the corresponding generalized Pauli group for each of the composite dimension.

We close with a comment on the group $P_{d}$. Such a group can be generated from the Weyl pair $(X, Z)$. Indeed, the group $P_{d}$ is a finite group of order $d^{3}$ with elements $q^{a} X^{b} Z^{c}$ (where $a, b, c \in \mathbf{Z}_{d}$ ) and matrix multiplication for group law. It is a subgroup of the unitary group $\mathrm{U}_{d}$. The normaliser of $P_{d}$ in $\mathrm{U}_{d}$ is a Clifford-type group in $d$ dimensions noted $C_{d}$. More precisely, $C_{d}$ is the set $\{U \in$ $\left.\mathrm{U}_{d} \mid U P_{d} U^{\dagger}=P_{d}\right\}$ endowed with matrix multiplication. The Pauli group $P_{d}$ as well as any other invariant subgroup of $C_{d}$ can be used for stabilizing errors in quantum computing. These concepts are very important in the case of n-qubit systems (corresponding to $d=2^{n}$ ). These matters, in connection with the decomposition of $P_{2^{n}}$ in terms of $P_{2}$, are the object of a future work.

\section{ACKNOWLEDGEMENTS}

The present paper is based on an invited talk to the International Workshop on New Trends in Science and Technology (3-4 November 2008, Ankara, Turkey). The author thanks the organizers for making possible this interesting pluri-disciplinary workshop.

\section{REFERENCES}

[1] M. Daoud, Y. Hassouni, and M. Kibler. The $k$ fermions as objects interpolating between fermions and bosons. In B. Gruber and M. Ramek, editors, Symmetries in Science X, pages 63-67. Plenum Press, New York, 1998, arXiv:quant-ph/9710016.

[2] M. Daoud, Y. Hassouni, and M. Kibler. Generalized supercoherent states. Phys. Atom. Nuclei, 61:18211824, 1998, arXiv:quant-ph/9804046.

[3] M. Daoud and M. Kibler. Fractional supersymmetric quantum mechanics as a set of replicas of ordinary supersymmetric quantum mechanics. Phys. Lett. A, 321:147-151, 2004, arXiv:math-ph/0312019.

[4] M. Daoud and M.R. Kibler. Fractional supersymmetry and hierarchy of shape invariant potentials. J. Math. Phys., 47:122108 (11 pages), 2006, arXiv:quant-ph/0609017.

[5] M.R. Kibler. On the Wigner-Racah algebra of the group $S U_{2}$ in a non-standard basis. In T. Lulek, B. Lulek, and A. Wal, editors, Symmetry and Structural Properties of Condensed Matter, pages 222233. World Scientific, Singapore, 1999, arXiv:math$\mathrm{ph} / 9810019$.

[6] M. Kibler and M. Daoud. Variations on a theme of quons: I. A non standard basis for the Wigner-Racah algebra of the group SU(2). Recent Res. Devel. Quantum Chem., 2:91-99, 2001, arXiv:physics/9712034.
[7] M.R. Kibler. Representation theory and WignerRacah algebra of the group $\mathrm{SU}(2)$ in a noncanonical basis. Collect. Czech. Chem. Commun., 70:771-796, 2005, arXiv:quant-ph/0504025.

[8] P. Delsarte, J.M. Goethals, and J.J. Seidel. Bounds for systems of lines and Jacobi polynomials. Philips Res. Repts., 30:91-105, 1975.

[9] I.D. Ivanović. Geometrical description of quantum state determination. J. Phys. A: Math. Gen., 14: 3241-3245, 1981.

[10] W.K. Wootters and B.D. Fields. Optimal statedetermination by mutually unbiased measurements. Ann. Phys. (N.Y.), 191:363-381, 1989.

[11] A.R. Calderbank, P.J. Cameron, W.M. Kantor, and J.J. Seidel. Zopf 4-Kerdock codes orthogonal spreads and extremal euclidean line-sets. Proc. London Math. Soc., 75:436-480, 1997.

[12] S. Bandyopadhyay, P.O. Boykin, V. Roychowdhury, and F. Vatan. A new proof for the existence of mutually unbiased bases. Algorithmica, 34:512-528, 2002, arXiv:quant-ph/0103162.

[13] J. Lawrence, Č. Brukner, and A. Zeilinger. Mutually unbiased binary observable sets on $N$ qubits. Phys. Rev. A, 65:032320 (5 pages), 2002.

[14] A. Klappenecker and M. Rötteler. Constructions of mutually unbiased bases. Lect. Notes Comput. Sci., 2948:137-144, 2004, arXiv:quant-ph/0309120.

[15] K.S. Gibbons, M.J. Hoffman, and W.K. Wootters. Discrete phase space based on finite fields. Phys. Rev. A, 70:062101 (23 pages), 2004.

[16] A.O. Pittenger and M.H. Rubin. Mutually unbiased bases, generalized spin matrices and separability. Linear Algebr. Appl., 390:255-278, 2004, arXiv:quant$\mathrm{ph} / 0308142$.

[17] M. Grassl. On SIC-POVMs and MUBs in Dimension 6. In J. Gruska, editor, Proceedings ERATO Conference on Quantum Information Science (EQIS 2004), pages 60-61. Tokyo, 2005, arXiv:quant-ph/0406175.

[18] M.R. Kibler. Angular momentum and mutually unbiased bases. Int. J. Mod. Phys. B, 20:1792-1801, 2006, arXiv:quant-ph/0510124.

[19] M.R. Kibler and M. Planat. A SU(2) recipe for mutually unbiased bases. Int. J. Mod. Phys. B, 20: 1802-1807, 2006, arXiv:quant-ph/0601092.

[20] M. Planat, M. Saniga, and M.R. Kibler. Quantum entanglement and projective ring geometry. SIGMA, 2: article 066 (14 pages), 2006, arXiv:quant-ph/0605239.

[21] O. Albouy and M.R. Kibler. SU $\mathrm{SU}_{2}$ nonstandard bases: case of mutually unbiased bases. SIGMA, 3:article 076 (22 pages), 2007, arXiv:quant-ph/0701230.

[22] M.R. Kibler. Miscellaneous applications of quons. SIGMA, 3:article 092 (14 pages), 2007, arXiv:0709.3698 (quant-ph).

[23] O. Albouy and M.R. Kibler. A unified approach to SIC-POVMs and MUBs. J. Russian Laser Res., 28: 429-438, 2007, arXiv:0704.0511 (quant-ph).

[24] M.R. Kibler. Variations on a theme of Heisenberg, Pauli and Weyl. J. Phys. A: Math. Theor., 41:375302 (19 pages), 2008, arXiv:0807.2837 (quant-ph).

[25] M. Planat and M. Kibler. Unitary reflection groups for quantum fault tolerance. arXiv:0807.3650 (quantph).

[26] O. Albouy. Unpublished, 2008. 